\title{
The timing of breast cancer surgery within the menstrual cycle
}

\author{
A.P. Corder, M. Cross, S.A. Julious ${ }^{1}$, M.A. Mullee ${ }^{1}$ and I. Taylor* \\ University Surgical Unit, ${ }^{1}$ Medical Statistics and Computing, Southampton General Hospital, \\ Southampton SO9 $4 X Y, U K$
}

\begin{abstract}
Summary: The effects of the timing of surgery, fine needle aspiration cytology (FNAC) and mammography within the menstrual cycle were investigated retrospectively in 211 premenopausal breast cancer patients. The day of surgery within the menstrual cycle was known for 157 women whose menstrual cycles were regular. Recurrence and survival were closely similar whether surgery was performed during days 3-12 or at other times (days 0-2 or 13-32) in the cycle. Outcome was also unrelated to the timing of mammography which was known in 101 cases. There were no significant differences in recurrence or survival associated with the timing of FNAC when all patients were considered.

In the subset of lymph node negative patients $(n=32)$, however, FNAC outside days 3-12 was associated with a significantly decreased rate of relapse $(R R=0.25,95 \% \mathrm{CI}=0.07-0.9)$ and death $(\mathbf{R R}=0.10,95 \% \mathbf{C I}=0.02-0.9)$. These patients, however, also had significantly smaller tumours (median $=2 \mathrm{~cm}$, interquartile range $=2-3 \mathrm{~cm}$ ) than patients having FNAC on days 3-12 of the cycle (median $=3$, interquartile range $=2-4)($ Mann-Whitney: $z=2.11, P=0.04)$.

We have not confirmed that surgery or FNAC during days 3-12 of the cycle is associated with a poorer outcome than interventions during other parts of the cycle.
\end{abstract}

\section{Introduction}

Many breast carcinomas contain hormone receptors ${ }^{1}$ which influence their behaviour. ${ }^{2}$ Recently, it has been proposed that the hormonal environment during breast cancer surgery may have a significant effect on outcome. ${ }^{3}$ Studies of recurrence and survival following breast cancer surgery performed at different points in the menstrual cycle have, however, yielded conflicting results. ${ }^{4-6}$ The importance of the timing of breast cancer surgery within the menstrual cycle remains highly controversial.

The most striking results yet obtained are those of Badwe et al. ${ }^{4}$ They found that patients undergoing breast cancer excision between days 3 and 12 of the menstrual cycle had very much poorer recurrence-free and overall survival than those undergoing excision at other times in the cycle. This effect was as important as lymph node status and was confined to lymph node positive patients. Because of the potential importance of these findings, we have investigated whether they can be reproduced using data from our own unit.

Correspondence: A.P. Corder, M.S., F.R.C.S., 31 Manor Park, Weston, Bath BA1 3RJ, UK.

*Present address: Department of Surgery, University College London, The Rayne Institute, 5 University Street, London WCIE 6JJ, UK.

Accepted: 1 July 1993

\section{Patients and methods}

We identified premenopausal patients undergoing breast cancer surgery between $29 / 6 / 1981$ and $12 / 1$ / 1988 using the records of the Southampton University Surgical Unit. These records included the date of surgery within the menstrual cycle and cycle details including regularity and duration. We also attempted to determine the timing of fine needle aspiration cytology (FNAC) and mammography within the menstrual cycle, using hospital notes and radiology records. Details of tumour pathology, staging, subsequent adjuvant therapy, recurrence and survival were obtained from the University Surgical Unit records.

Diagnoses were made by FNAC and mammography. Patients were treated by mastectomy and axillary clearance or wide local excision, axillary clearance and radiotherapy depending on tumour suitability and patient preference. Patients with tumour in 1-3 axillary nodes were offered adjuvant chemotherapy and those with greater than $75 \%$ axillary node involvement were offered adjuvant radiotherapy.

We identified 211 premenopausal women who underwent surgery for breast cancer. The day of surgery within the menstrual cycle was known for 157 women whose menstrual cycles were regular. We excluded patients with irregular cycles, those 
taking the oral contraceptive pill and patients who were pregnant. The timing of mammography was known in 101 cases and the timing of FNAC, in 61 cases.

Statistical analyses were carried out using the SAS statistical package. ${ }^{7}$ Initial survival analyses were performed with the LIFETEST procedure in SAS. The log-rank $\chi^{2}$ statistic was used to assess differences between groups. A Cox proportional hazards model, using the PHREG procedure, was undertaken to investigate the association between timing of breast cancer surgery and various prognostic factors.

\section{Results}

Fifty-eight patients underwent breast cancer excision on days 3-12 after the start of their last menstrual period. The remaining 99 patients had operations performed at other times in the menstrual cycle (days $0-2$ or $13-32$ ). Both recurrencefree and overall survival were virtually identical in these two groups of patients (log-rank $\chi^{2}=0.49$, d.f. $=1, \quad P=0.48$ for relapse and log-rank $\chi^{2}=0.75$, d.f. $=1, P=0.39$ for death rates) (Figures 1 and 2). The groups were similar in terms of nodal status ( $38 \%$ node positive days $3-12,44 \%$ node positive rest of cycle; Pearson $\chi^{2}=0.48$, d.f. $=1, P=0.49$ ).

We then examined outcome following any intervention including FNAC $(n=61)$ and mammography $(n=101)$. Patients having FNAC outside days $3-12$ of the cycle had somewhat decreased relapse $\left(\log\right.$-rank $\dot{\chi}^{2}=1.09$, d.f. $=1$, $P=0.30 ;$ relative tisk $(\mathbf{R R})=0.66,95 \%$ confidence interval $(\mathrm{CI})=0.32-1.38$ ) and death rates (log-rank $\chi^{2}=2.50$, d.f. $=1, P=0.11$,
$R R=0.49,95 \% C I=0.2-1.22)$. These differences did not reach conventional statistical significance.

In the subset of lymph node negative patients $(n=32)$, however, relapse and death rates were significantly lower in those having FNAC outside days $3-12$ of the cycle $(n=21)$ (RR for relapse $=0.25,95 \% \quad C I=0.07-0.9 ; \quad R R$ for death $=0.10,95 \% \mathrm{CI}=0.02-0.9)($ Figures 3 and 4). These patients, however, also had significantly smaller tumours (median $=2 \mathrm{~cm}$, interquartile range $=2-3 \mathrm{~cm}$ ) than patients having operations on days 3-12 of the cycle (median $=3$, interquartile range $=2-4) \quad($ Mann-Whitney: $z=2.11$, $P=0.04$ ). Tumour size did not reach significance as an independent prognostic factor in this study. The groups were well matched for oestrogen receptor status and tumour type.

Relapse and death rates of patients having mammography during days 3-12 of the cycle were similar to those of patients having mammography at other times in the cycle $\left(\log -\operatorname{rank} \chi^{2}=0.11\right.$, d.f. $=1, P=0.74$ for relapse and log-rank $\chi^{2}=0.40$, d.f. $=1, P=0.53$ for death rates).

\section{Discusaion}

In contrast to Badwe et al. ${ }^{4}$, we were unable to show that the results of surgery for breast cancer are related to the timing of surgery within the menstrual cycle. Similar negative results have been reported by a majority of investigators. ${ }^{5,6,8-10}$ Other investigators have, however, independently published results similar to those of Badwe et al. though none have shown such a large effect." Despite our negative findings, it may therefort be premature to dismiss the possibility completely that the timing of breast cancer surgery within the menarual cycle might affect outcome.

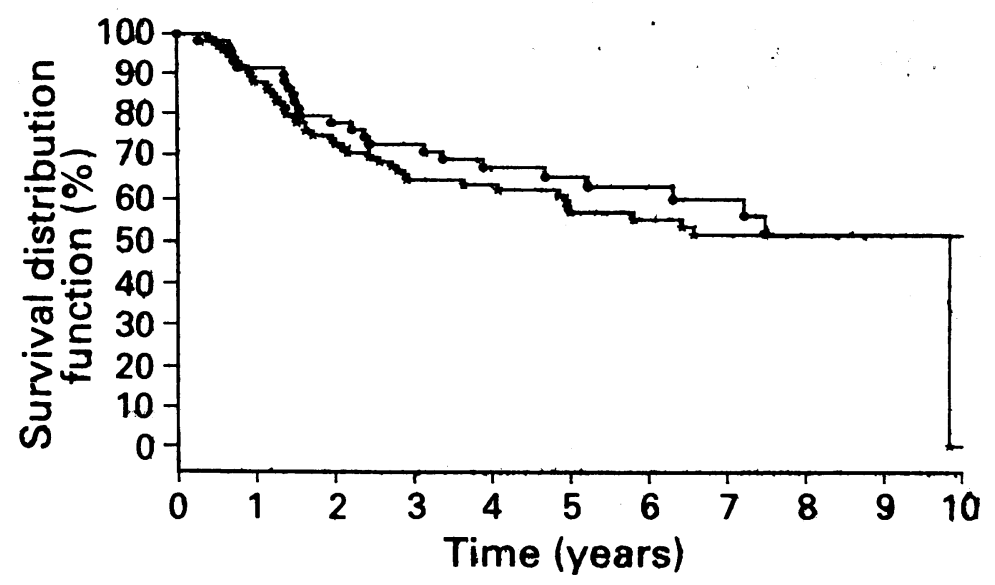

Figure 1 Disease-free survival by time of surgery in the menstrual cycle. 0 , days $3-12$; $\star$, days $0-2$ and $13-32$. 


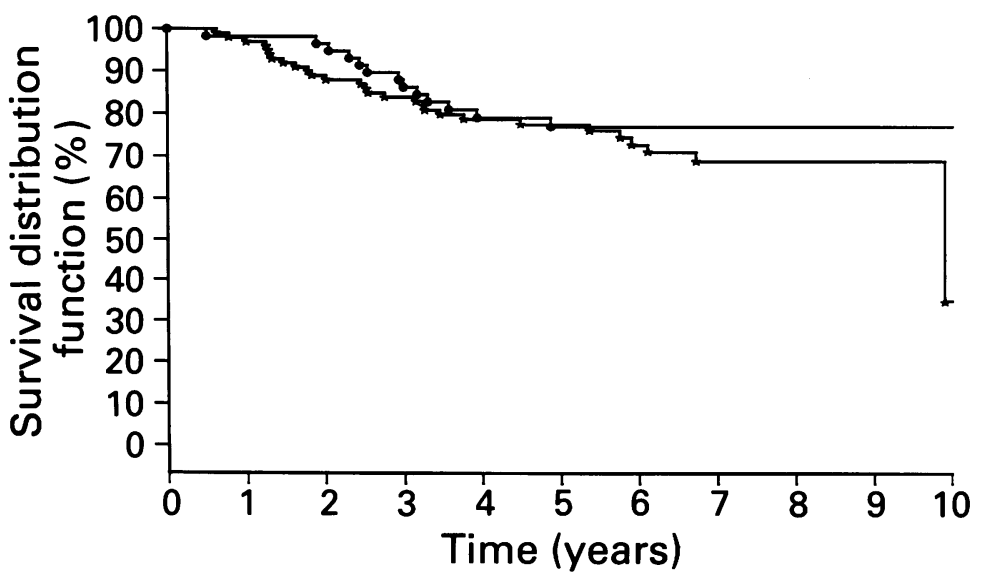

Figure 2 Overall survival by time of surgery in the menstrual cycle. 0 , days $3-12$; $\star$, days $0-2$ and $13-32$.

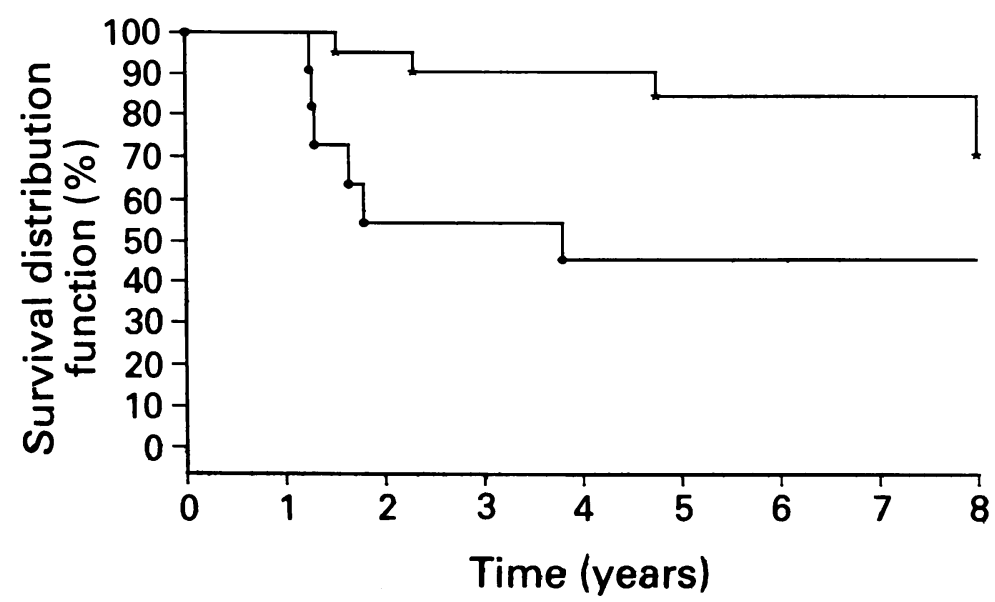

Figane 3 Disease-free survival of lymph node negative patients by time of fine needle aspiration cytology in the menstrual cycle. , days $3-12 ; \star$, days $0-2$ and $13-32$.

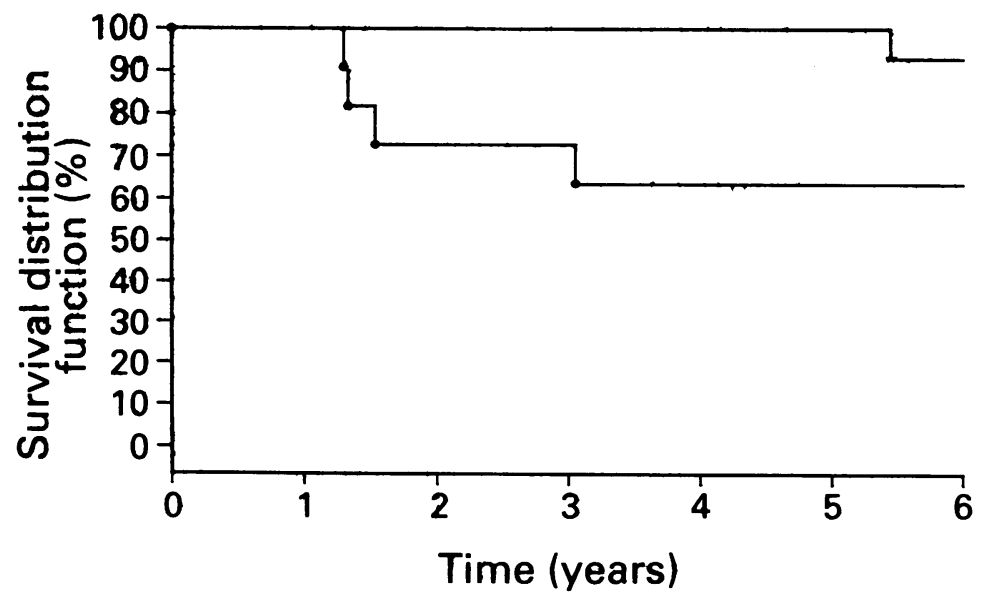

Figure 4 Overall survival of lymph node negative patients by time of fine needle aspiration cytology in the menstrual cycle. , days $3-12 ; \star$, days $0-12$ and $13-32$. 
In response to a personal communication from Badwe and colleagues, we also investigated the possible effects of the timing of FNAC and mammography. Outcome appeared to be unrelated to the timing of mammography but there was a trend towards poorer recurrence-free and overall survival in patients having FNAC on days $3-12$ of the cycle. This effect was statistically significant in lymph node negative patients but was not seen in those who were lymph node positive. However, tumours were significantly larger in lymph node negative patients having FNAC on days 3-12 of the cycle. Tumour size is an accepted prognostic factor, ${ }^{12}$ although it failed to reached significance as an independent prognostic factor in this study, probably because of study size. A chance difference in tumour size may be the explanation of the apparent influence of timing of FNAC within the lymph node negative patients. The effect of timing of breast cancer excision appeared to be limited to

\section{References}

1. Margreiter, R. \& Wiegele, J. Tamoxifen (Nolvadex) for premenopausal patients with advanced breast cancer. Breast Cancer Res Treat 1984, 4: 45-48.

2. Buchanan, R.B., Blamey, R.W., Durrant, K.R. et al. A randomised comparison of tamoxifen with surgical oophorectomy in premenopausal patients with advanced breast cancer. J Clin Oncol 1986, 4: 1326-1330.

3. Hruschesky, J.M., Bluming, A.Z., Gruber, S.A. \& Sothern, R.B. Menstrual influence on surgical cure of breast cancer. Lancet 1989, 2: 949-952.

4. Badwe, R.A., Gregory, W.M., Chaudary, M.A. et al. Timing of surgery during menstrual cycle and survival of premenopausal women with operable breast cancer. Lancet 1991, 337: $1261-1264$.

5. Low, S.C., Galea, M.H. \& Blamey, R.W. Timing breast cancer surgery (letter). Lancet 1991, 338: 691.

6. Powles, T.J., Ashley, S.E., Nash, A.G., Tidy, A., Gazet, J.C. \& Ford, H.T. Menstrual surgical timing (letter). Lancet 1991, 337: 1604.

7. SAS User's Guide: Statistics, Version 6, 4th edn. Cary, North Carolina, SAS Institute, Inc., 1990.

8. Goldhirsch, A., Belber, R.D., Forbes, J. et al. Timing breast cancer surgery (letter). Lancet 1991, 338: 691-692. lymph node positive patients in the studies of Badwe et al. ${ }^{4}$ and of Senie et al. ${ }^{11}$

It has been suggested that an effect due to FNAC might explain the different findings of studies which have investigated the effect of timing of breast cancer surgery. ${ }^{13}$ It has also been suggested that the effect of timing of breast cancer surgery should be investigated prospectively and recruitment to such a study is currently under way. ${ }^{14}$ On our unit and on others, it is usual for breast cancer patients to undergo FNAC and mammography at their first out-patient visit. They return the week after for results and counselling and then undergo definitive surgery the next week. Usually, therefore, there is a 2 week gap between FNAC and surgery, placing one in a possible high risk period and the other in a low risk period or vice versa. Units undertaking prospective study of this issue will need to address this point and may have to modify the timing of their diagnostic and therapeutic procedures.

9. Rageth, J.C., Wyss, P., Unger, C. \& Hochuli, E. Timing of breast cancer surgery within the menstrual cycle: influence on lymph node involvement, receptor status, postoperative metastatic spread and local recurrence. Ann Oncol 1991, 2: 269-272.

10. Nathan, B., Bates, T., Anbazhagan, R. \& Norman, A.R. Timing of surgery for breast cancer in relation to the menstrual cycle and survival of premenopausal women. $\mathrm{Br} J$ Surg 1993, 80: 43.

11. Senie, R.T., Rosen, P.P., Rhodes, P. \& Lesser, M.L. Timing of breast cancer excision during the menstrual cycle influences duration of disease free survival. Ann Intern Med 1991, 115: 337-342.

12. Haybittle, J.L., Blamey, R.W., Elston, C.W. et al. A prognostic index in primary breast cancer. $B r J$ Cancer 1982, 45: 361-366.

13. Gregory, W.M., Richards, M.A. \& Fentiman, I.S. Optimal timing of initial breast cancer surgery (letter). Ann Intern Med 1992, 116: 268-269.

14. Sainsbury, J.R.C. \& Round, C.R. Timing of surgery for breast cancer and menstrual cycle (abstract). The Breast 1992, 1: 152 . 